\title{
Self-alignment algorithm for strapdown inertial navigation system under strong flurry interference
}

\author{
WANG Yuegang \\ Department of Automatic Control Engineering \\ Xi'an Research Institute of High technology \\ Xi'an, 710025 \\ e-mail: wangyueg@163.com
}

\author{
YANG Jiasheng \\ Department of Automatic Control Engineering \\ Xi'an Research Institute of High technology \\ Xi'an, 710025 \\ e-mail: 378769761@qq.com
}

\begin{abstract}
For the strong flurry interrupting, the body will suffer large swaying motion when it is in erecting state the output of its strapdown inertial navigation system (SINS) will be disturbed for the high gravitational center of IMU, the conventional methods are difficult to achieve alignment rapidly and accurately, to solve this problem, an antiinterference self-alignment algorithm for SINS which under strong flurry is presented, which utilizes the continuous attitude update in inertial reference frame to record the attitude changes caused by sway interrupt to remove the angular interrupting, and uses the characteristics that the body exists a shake center whose speed is zero to remove the linear movement interrupting by acquiring the equivalent specific force of the shake center, and then uses the estimation of the initial attitude to determinate the attitude of the body. The simulation result show that the presented algorithm can accomplish alignment quickly even in the presence of strong flurry interference without coarse alignment phase.
\end{abstract}

Keywords- inertial navigation system; interference; selfalignment; strong flurry

\section{INTRODUCTION}

The initial alignment process is required before the strapdown inertial navigation system (SINS) starts navigation, however, for the strong flurry interrupting ,the body suffers large swaying motion when its in erecting state, the outputs of SINS are disturbed for its high gravitational center, the conventional alignment methods are difficult to achieve alignment rapidly and accurately, to solve this problem, article in [1] presents a coarse alignment method which utilizes the gravity vector in the inertial frame, and its performance is verified by turntable-test [2], this method can circumvent the influence of angular swing interference theoretically, however, it can not suppress linear motion interference effectively; [3] presents a gravity-based antiinterference coarse alignment algorithm which uses the gravity vector in the inertial frame and the LMS curve fitting algorithm to fit linear motion interference-contaminated reference vectors; article in [4] utilizes the IIR low-pass filter to filter out the angular and acceleration interference in inertial frame, and then the coarse alignment of rotary SINS is achieved. The above mentioned methods are coarse alignment algorithm, when they are finished,the fine alignment is needed to fulfill the alignment accuracy requirements; the alignment schemes are both presented in
[5-6] which use the low-pass filter(IIR or FIR filter) to extract the reference vector in inertial frame, these alignment schemes can suppress the angular and linear motion interference to some extent, nevertheless, the strong flurry disturbance causes the SINS rock with low frequency and large amplitude [7], which make it is hard to set cut-off frequency to filter out the disturbance completely, when it come to Wavelet De-Noising method [8], the above problem is also existed, and its implementation is complexity.

For the self-alignment of SINS under strong flurry, it is need to remove effects caused by the angular swing interference and linear motion interference. When the body is in erecting state, there is no relative motion between the body and the ground, though the body will sway under the strong flurry interference, there is a shake center with zero velocity, therefore, if the accelerator equivalent specific force outputs can be obtained at shake center, the linear motion interference can be removed, and if the body can deemed as rigid body, the attitudes of the different points of the body are the same, therefore, we achieve alignment by obtaining the attitude of the shake center.

Therefore, to remove the linear motion interference, the solution idea is using the lever arm effects to get the equivalent specific force outputs at the shake center, and then using them in the later alignment operation; to remove the angular swing interference, the solution idea is using the continuous attitude update in inertial frame to record the attitude changes caused by sway interrupt, and then use the optimal estimation of initial attitude to achieve initial alignment.

\section{SELF-ALIGNMENT ALGORITHM FOR ANGULAR SWING INTERFERENCE}

The coordinate frames used in this paper include navigation coordinate frame (n-frame), body coordinate frame (b-frame), Earth-fixed coordinate frame (e-frame), inertial coordinate frame ( $\mathrm{i}$-frame) et al, the specific meaning of these coordinate frames can be seen in [9].

To remove the angular swing interference, the solution idea is using the attitude update in inertial frame to record the attitude changes caused by sway interrupt, at the same time, the initial attitude is estimated simultaneously, and then combine the above two results to finish the initial alignment. The principle of the algorithm as follows. 
At the moment of the alignment begins, for the i-frame and b-frame are in superposition, we have $\boldsymbol{q}_{\mathrm{i}}^{\mathrm{b}}(0)=\left[\begin{array}{llll}1 & 0 & 0 & 0\end{array}\right]^{\mathrm{T}}$, according to the quaternion multiplying operation theorem, we have

$$
\begin{aligned}
\boldsymbol{q}_{\mathrm{n}}^{\mathrm{b}}(t) & =\boldsymbol{q}_{\mathrm{i}}^{\mathrm{b}}(t) \otimes \boldsymbol{q}_{\mathrm{n}}^{\mathrm{i}}(t) \\
& =\boldsymbol{q}_{\mathrm{i}}^{\mathrm{b}}(0, t) \otimes \boldsymbol{q}_{\mathrm{i}}^{\mathrm{b}}(0) \otimes \boldsymbol{q}_{\mathrm{n}}^{\mathrm{i}}(t) \\
& =\boldsymbol{q}_{\mathrm{i}}^{\mathrm{b}}(0, t) \otimes \boldsymbol{q}_{\mathrm{i}}^{\mathrm{b}}(0) \otimes \boldsymbol{q}_{\mathrm{n}}^{\mathrm{i}}(0, t) \otimes \boldsymbol{q}_{\mathrm{n}}^{\mathrm{i}}(0) \\
& =\boldsymbol{q}_{\mathrm{i}}^{\mathrm{b}}(0, t) \otimes \boldsymbol{q}_{\mathrm{i}}^{\mathrm{b}}(0) \otimes \boldsymbol{q}_{\mathrm{n}}^{\mathrm{i}}(0, t) \otimes \boldsymbol{q}_{\mathrm{b}}^{\mathrm{i}}(0) \otimes \boldsymbol{q}_{\mathrm{n}}^{\mathrm{b}}(0) \\
& =\boldsymbol{q}_{\mathrm{i}}^{\mathrm{b}}(0, t) \otimes \boldsymbol{q}_{\mathrm{n}}^{\mathrm{i}}(0, t) \otimes \boldsymbol{q}_{\mathrm{n}}^{\mathrm{b}}(0)
\end{aligned}
$$

In the above, $\boldsymbol{q}_{\mathrm{n}}^{\mathrm{i}}(0)$ representing the orientation of the $\mathrm{i}$ frame relative to the $\mathrm{n}$-frame at $\mathrm{t}=0, \boldsymbol{q}_{\mathrm{i}}^{\mathrm{n}}(0, t)$ representing the changes of $\boldsymbol{q}_{\mathrm{i}}^{\mathrm{n}}$ from $\mathrm{t}=0$ to time $\mathrm{t}, \boldsymbol{q}_{\mathrm{i}}^{\mathrm{b}}(0, t)$ representing the changes of $\boldsymbol{q}_{\mathrm{i}}^{\mathrm{b}}$ from $\mathrm{t}=0$ to time $\mathrm{t}$, thus the $\boldsymbol{q}_{\mathrm{i}}^{\mathrm{n}}(0, t)$ and $\boldsymbol{q}_{\mathrm{i}}^{\mathrm{b}}(0, t)$ is initialized to:

$$
\boldsymbol{q}_{\mathrm{i}}^{\mathrm{n}}(0,0)=\boldsymbol{q}_{\mathrm{i}}^{\mathrm{b}}(0,0)=\left[\begin{array}{llll}
1 & 0 & 0 & 0
\end{array}\right]^{\mathrm{T}}
$$

$\boldsymbol{q}_{\mathrm{i}}^{\mathrm{b}}(0, t), \quad \boldsymbol{q}_{\mathrm{i}}^{\mathrm{n}}(0, t)$ can be obtained by solving the following differential equation

$$
\begin{aligned}
& \dot{\boldsymbol{q}}_{\mathrm{i}}^{\mathrm{b}}(0, t)=1 / 2 \boldsymbol{q}_{\mathrm{i}}^{\mathrm{b}}(0, t) \otimes \omega_{\mathrm{bi}}^{\mathrm{i}} \\
& \dot{\boldsymbol{q}}_{\mathrm{i}}^{\mathrm{n}}(0, t)=1 / 2 \boldsymbol{q}_{\mathrm{i}}^{\mathrm{n}}(0, t) \otimes \omega_{\mathrm{ni}}^{\mathrm{i}}
\end{aligned}
$$

According to specific force equation

$$
\dot{V}_{\mathrm{en}}^{\mathrm{n}}=\boldsymbol{f}^{\mathrm{n}}-\left(2 \omega_{\mathrm{ie}}^{\mathrm{n}}+\omega_{\mathrm{en}}^{\mathrm{n}}\right) \times \boldsymbol{V}_{\mathrm{en}}^{\mathrm{n}}+\boldsymbol{g}^{\mathrm{n}}
$$

Thus we can get

$$
\begin{aligned}
& \left(\boldsymbol{q}_{\mathrm{b}}^{\mathrm{n}}(0)\right)^{*} \otimes\left(\boldsymbol{q}_{\mathrm{i}}^{\mathrm{n}}(0, t)\right)^{*} \otimes\left[\dot{\boldsymbol{V}}_{\mathrm{en}}^{\mathrm{n}}+\left(2 \boldsymbol{\omega}_{\mathrm{le}}^{\mathrm{n}}+\boldsymbol{\omega}_{\mathrm{en}}^{\mathrm{n}}\right) \times \boldsymbol{V}_{\mathrm{en}}^{\mathrm{n}}-\right. \\
& \left.\boldsymbol{g}^{\mathrm{n}}\right] \otimes \boldsymbol{q}_{\mathrm{i}}^{\mathrm{n}}(0, t) \otimes \boldsymbol{q}_{\mathrm{b}}^{\mathrm{n}}(0)=\left(\boldsymbol{q}_{\mathrm{i}}^{\mathrm{b}}(0, t)\right)^{*} \otimes \boldsymbol{f}^{\mathrm{b}} \otimes \boldsymbol{q}_{\mathrm{i}}^{\mathrm{b}}(0, t)
\end{aligned}
$$

The following definitions can be made

$$
\begin{aligned}
\boldsymbol{\beta}(t)= & \left(\boldsymbol{q}_{\mathrm{i}}^{\mathrm{b}}(0, t)\right)^{*} \otimes \boldsymbol{f}^{\mathrm{b}} \otimes \boldsymbol{q}_{\mathrm{i}}^{\mathrm{b}}(0, t) \\
\boldsymbol{\alpha}(t)= & \left(\boldsymbol{q}_{\mathrm{i}}^{\mathrm{n}}(0, t)\right)^{*} \otimes\left[\dot{\boldsymbol{V}}_{\mathrm{en}}^{\mathrm{n}}+\right. \\
& \left.\left(2 \boldsymbol{\omega}_{\mathrm{ie}}^{\mathrm{n}}+\boldsymbol{\omega}_{\mathrm{en}}^{\mathrm{n}}\right) \times \boldsymbol{V}_{\mathrm{en}}^{\mathrm{n}}-\boldsymbol{g}^{\mathrm{n}}\right] \otimes \boldsymbol{q}_{\mathrm{i}}^{\mathrm{n}}(0, t)
\end{aligned}
$$

According to equation (3), (4) and (5), the following equation can be obtained

$$
\boldsymbol{q}_{\mathrm{b}}^{\mathrm{n}}(0) \otimes \boldsymbol{\beta}(t)-\boldsymbol{\alpha}(t) \otimes \boldsymbol{q}_{\mathrm{b}}^{\mathrm{n}}(0)=\mathbf{0}
$$

Defining $\boldsymbol{M}(\boldsymbol{\beta}(t))$ and $\boldsymbol{M}(\boldsymbol{\alpha}(t))$ as follows

$$
\boldsymbol{M}(\boldsymbol{\beta}(t))=\left[\begin{array}{cc}
0 & \boldsymbol{\beta}^{\mathrm{T}} \\
\boldsymbol{\beta} & -\boldsymbol{\beta} \times
\end{array}\right], \quad \boldsymbol{M}(\boldsymbol{\alpha}(t))=\left[\begin{array}{cc}
0 & -\boldsymbol{\alpha}^{\mathrm{T}} \\
\boldsymbol{\alpha} & \boldsymbol{\alpha} \times
\end{array}\right]
$$

Where $\beta \times$ is the skew symmetric matrix of the vector $\beta$, $\alpha \times$ is the skew symmetric matrix of the vector, according to the above definition, the equation (6) can be written as

$$
(\boldsymbol{M}(\boldsymbol{\beta}(t))-\boldsymbol{M}(\boldsymbol{\alpha}(t))) \boldsymbol{q}_{\mathrm{b}}^{\mathrm{n}}(0)=\mathbf{0}
$$

Thus, the alignment problem is now translated into the famous Wahba attitude determination problem [10] as follows:

$$
\begin{aligned}
& \min \int_{0}^{t}\left\|(\boldsymbol{M}(\boldsymbol{\beta}(t))-\boldsymbol{M}(\boldsymbol{\alpha}(t))) \boldsymbol{q}_{\mathrm{b}}^{\mathrm{n}}(0)\right\| d t \\
& =\left(\boldsymbol{q}_{\mathrm{b}}^{\mathrm{n}}(0)\right)^{\mathrm{T}} \int_{0}^{t}(\boldsymbol{M}(\boldsymbol{\beta}(t))-\boldsymbol{M}(\boldsymbol{\alpha}(t)))^{\mathrm{T}}(\boldsymbol{M}(\boldsymbol{\beta}(t))-\boldsymbol{M}(\boldsymbol{\alpha}(t))) d t \boldsymbol{q}_{\mathrm{b}}^{\mathrm{n}}(0) \\
& =\min _{\boldsymbol{q}_{\mathrm{b}}^{\mathrm{n}}}\left(\boldsymbol{q}_{\mathrm{b}}^{\mathrm{n}}(0)\right)^{\mathrm{T}} \boldsymbol{K} \boldsymbol{q}_{\mathrm{b}}^{\mathrm{n}}(0)
\end{aligned}
$$

Subject to: $\left(\boldsymbol{q}_{\mathrm{b}}^{\mathrm{n}}(0)\right)^{\mathrm{T}} \boldsymbol{q}_{\mathrm{b}}^{\mathrm{n}}(0)=1$

Where

$$
\boldsymbol{K}=\int_{0}^{t}(\boldsymbol{M}(\boldsymbol{\beta}(t))-\boldsymbol{M}(\boldsymbol{\alpha}(t)))^{\mathrm{T}}(\boldsymbol{M}(\boldsymbol{\beta}(t))-\boldsymbol{M}(\boldsymbol{\alpha}(t))) d t
$$

The minimization will be achieved if only $\boldsymbol{q}_{\mathrm{b}}^{\mathrm{n}}(0)$ is chosen to be the normalized eigenvector corresponding to the smallest eigenvalue of matrix $\mathrm{K}$, the derivation process is provided in [9].

\section{ANALYSIS OF THE ABOVE SELF-ALIGNMENT ALGORITHM}

According to self-alignment algorithm in section 1, if the body makes the angular swing only, and there is no linear motion, we can get that $\dot{\boldsymbol{V}}_{\mathrm{en}}^{\mathrm{n}}=0, \boldsymbol{V}_{\mathrm{en}}^{\mathrm{n}}=0, \tilde{\boldsymbol{f}}_{\mathrm{sf}}^{\mathrm{n}}=-\boldsymbol{g}^{\mathrm{n}}$ through specific force equation, thus $\boldsymbol{\beta}(t)$ can be obtained as follows.

$$
\begin{aligned}
\boldsymbol{\beta}(t) & =\left(\boldsymbol{q}_{\mathrm{i}}^{\mathrm{b}}(0, t)\right)^{*} \otimes \boldsymbol{f}^{\mathrm{b}} \otimes \boldsymbol{q}_{\mathrm{i}}^{\mathrm{b}}(0, t) \\
& =\left(\boldsymbol{q}_{\mathrm{i}}^{\mathrm{b}}(0, t)\right)^{*} \otimes\left(\tilde{\boldsymbol{f}}_{s f}^{\mathrm{b}}\right) \otimes \boldsymbol{q}_{\mathrm{i}}^{\mathrm{b}}(0, t)
\end{aligned}
$$

When the body suffers linear motion interference, the following equation can be obtained.

$$
\boldsymbol{f}_{\mathrm{sf}}^{\mathrm{n}}=\tilde{\boldsymbol{f}}_{\mathrm{sf}}^{\mathrm{n}}+\tilde{\boldsymbol{f}}_{D}^{\mathrm{n}}=\dot{\boldsymbol{V}}_{\mathrm{en}}^{\mathrm{n}}+\left(2 \boldsymbol{\omega}_{\mathrm{ie}}^{\mathrm{n}}+\boldsymbol{\omega}_{\mathrm{en}}^{\mathrm{n}}\right) \times \boldsymbol{V}_{\mathrm{en}}^{\mathrm{n}}-\boldsymbol{g}^{\mathrm{n}}(9)
$$

from equation (9), it can be seen that the linear motion interference $\dot{\boldsymbol{V}}_{\mathrm{en}}^{\mathrm{n}}+\left(2 \boldsymbol{\omega}_{\mathrm{ie}}^{\mathrm{n}}+\boldsymbol{\omega}_{\mathrm{en}}^{\mathrm{n}}\right) \times \boldsymbol{V}_{\mathrm{en}}^{\mathrm{n}}$ make the additional specific force output $\tilde{\boldsymbol{f}}_{D}^{\text {n }}$, generally, it is hard to measure $\dot{\boldsymbol{V}}_{\text {en }}^{\mathrm{n}}$ and $\boldsymbol{V}_{\mathrm{en}}^{\mathrm{n}}$, thus they are be ignored only, that will lead extra alignment errors. When the body is in erecting state, there is no relative motion between the body and the ground, though the body will sway under the strong flurry interference, there is a shake center with zero velocity, in the shake center, $\dot{\boldsymbol{V}}_{\mathrm{en}}^{\mathrm{n}}=0, \boldsymbol{V}_{\mathrm{en}}^{\mathrm{n}}=0$, that is to say that if we can get the equivalent specific force outputs at the shake center and then use the alignment algorithm in section $\Pi$, we can remove the linear motion interference and the angular swing interference simultaneously.

\section{THE EQUIVALENT SPECIFIC FORCE OUTPUTS AT THE} SHAKE CENTER

The body's shake center is not the same as the SINS installation position usually, using the gyro output and the lever arm between the shake center and the SINS position, the equivalent specific force outputs at the shake center can be obtain through the lever arm effects. Suppose there is 
also a SINS in shake center also, it's specific force output and the origin SINS specific force output have the following relationship [11]

$$
\begin{aligned}
\boldsymbol{a}_{S}^{S}= & \boldsymbol{C}_{C}^{S}\left[\boldsymbol{a}_{C}^{C}+\boldsymbol{\omega}_{i C}^{C} \times \boldsymbol{\omega}_{i C}^{C} \times \boldsymbol{r}^{C}+\right. \\
& \left.\dot{\boldsymbol{\omega}}_{i C}^{C} \times \boldsymbol{r}^{C}+2\left(\boldsymbol{\omega}_{i C}^{C} \times \dot{\boldsymbol{r}}^{C}\right)+\ddot{\boldsymbol{r}}^{C}\right]
\end{aligned}
$$

Where $\boldsymbol{a}_{S}^{S}$ is specific force of the origin SINS, $\boldsymbol{a}_{C}^{C}, \boldsymbol{\omega}_{i C}^{C}$ are the specific force and gyro output of the shake center SINS respectively.

When the coordinate frames of shake center's SINS and the origin SINS are both choose as the b-frame, one the assumption that the body is rigid body and there is no relative position changes between the two SINS, we have

$$
\boldsymbol{a}^{C}=\boldsymbol{a}^{S}-\boldsymbol{\omega}_{i b}^{b} \times \boldsymbol{\omega}_{i b}^{b} \times \boldsymbol{r}^{b}-\dot{\boldsymbol{\omega}}_{i b}^{b} \times \boldsymbol{r}^{b}
$$

From equation (11), it can be conclude that the equivalent specific force outputs at the shake center can be calculated if the lever arm, the specific force and gyro output of the origin SINS, the angular acceleration are all known, however, the angular acceleration $\dot{\boldsymbol{\omega}}_{i b}^{b}$ can not be obtained directly, it need to be extracted from gyro output, if we difference two the gyro outputs directly, the noise will be magnified greatly for the measurement noise. To suppress the difference noise, the Tracking Differentiator (TD) [12] is design to compute $\dot{\boldsymbol{\omega}}_{i b}^{b}$, Fig. 1 shows its principle.

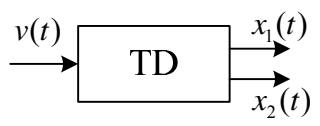

Figure 1. the block diagram of Tracking Differentiator

In the above, $x_{1}$ is the tracking signal of $v$, and $x_{2}$ is the differential signal of $v$, they can be calculated by the following formula.

$$
\left\{\begin{array}{l}
x_{1}(k+1)=x_{1}(k)+h x_{2}(k) \\
x_{2}(k+1)=x_{1}(k)+h f h a n\left(x_{1}(k)-v(k), x_{2}(k), r, h\right)
\end{array}\right.
$$

Where fhan $\left(x_{1}(k), x_{2}(k), r, h\right)$ is the fastest synthesis function.

Generally, the lever arm $\boldsymbol{r}^{b}$ between the shake center and the origin SINS can be measured previously through experiments, using the formula (11) and the TD filter, the equivalent specific force outputs at the shake center can be calculated.

\section{ALGORITHM IMPLEMENTATION}

The self-alignment algorithm for SINS under strong flurry is summarized as follows.

Step 1: initialize $\tilde{r}^{b} 、 \boldsymbol{q}_{i}^{n}(0,0) 、 \boldsymbol{q}_{i}^{b}(0,0)$;

Step 2: compute $\dot{\boldsymbol{\omega}}_{\boldsymbol{i} \boldsymbol{b}}^{\boldsymbol{b}}$ through TD filter

Step 3: compute the equivalent specific force outputs $\boldsymbol{f}_{C}^{b}$ at the shake center through equation (11);

Step 4: update $\boldsymbol{q}_{i}^{\boldsymbol{b}}(0, t), \boldsymbol{q}_{i}^{\boldsymbol{n}}(0, t)$ by solving equation (2);
Step 5: set $\boldsymbol{V}_{e n}^{n}=\dot{V}_{e n}^{n}=\mathbf{0}$, compute $\boldsymbol{\alpha}(t)$ and $\boldsymbol{\beta}(t)$ according to equation (4),(5) by using $\boldsymbol{f}_{C}^{\boldsymbol{b}}$ and $\boldsymbol{\omega}_{i \boldsymbol{b}}^{\boldsymbol{b}}$;

Step 6: determine $\tilde{\boldsymbol{q}}_{b}^{n}(0)$ by calculating the normalized eigenvector of $\mathrm{K}$ matrix belonging to the smallest eigenvalue;

Step 7: calculate $\boldsymbol{q}_{\boldsymbol{n}}^{\boldsymbol{b}}(t)$ through equation (1);

Step 8: go to step 2 if the error of $\tilde{\boldsymbol{q}}_{\boldsymbol{b}}^{n}(0)$ in two adjacent time small than error tolerance and until the end.

Step 9: finish alignment and start navigation.

\section{SIMULATION RESULTS AND DISCUSSIONS}

\section{A. Parameters used for simulation}

The body is assumed to be located at latitude $34^{\circ}(\mathrm{N})$ and longitude $110^{\circ}(\mathrm{E})$, the gyroscopes with drift rate $0.02^{\circ} / \mathrm{h}$ and accelerometers with bias $100 \mu \mathrm{g}$, the noise standard deviations are $0.01^{\circ} / \mathrm{h}$ and $50 \mu \mathrm{g}$ for gyroscopes and accelerometers, respectively, lever arm $\boldsymbol{r}^{\mathrm{b}}=[0.5 ; 5 ; 0.2]$, for the strong flurry interrupting, the body will suffer large swaying motion, owing to the uncertainty of wind direction, the most complex situation is that the body swing in three axis (roll axis ,pitch axis, yaw axis), which is reflected in the attitude changes, specifically, the different axis suffers different sway, we describe the body swing by the roll angle $\theta$,pitch angle $\gamma$ and yaw angle $\psi$ changes as follows.

$$
\left[\begin{array}{c}
\theta \\
\gamma \\
\psi
\end{array}\right]=\left[\begin{array}{l}
90+7^{\circ} \cos (0.3 \pi t) \\
5+6^{\circ} \cos \left(0.4 \pi t+\frac{2}{3} \pi\right) \\
30^{\circ}+5^{\circ} \cos \left(0.5 \pi t+\frac{4}{3} \pi\right)
\end{array}\right]+\left[\begin{array}{l}
\theta_{\text {randn }} \\
\gamma_{\text {randn }} \\
\psi_{\text {randn }}
\end{array}\right]
$$

where $\theta_{\text {randn }}, \gamma_{\text {randn }}$ and $\psi_{\text {randn }}$ are white noise with the means are $0^{\circ}$ and the standard deviations are $0.5^{\circ}, 1^{\circ}, 0.25^{\circ}$ respectively.

\section{B. results discussion}

According to the parameters setting in part A of section VI, the alignment algorithm is implemented directly without the coarse alignment phase, the simulation time is 280 seconds, the navigation solution of velocity which using true initialization is exhibited in Fig.3.

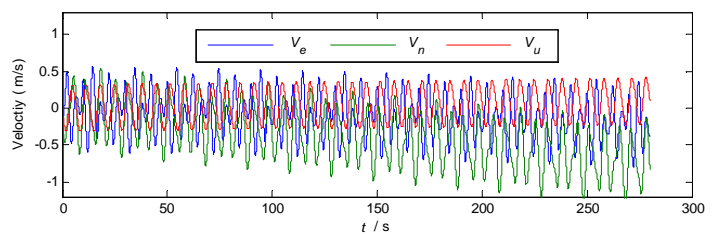

Figure 2. the velocity navigation result of SINS under flurry interference

Form the equation (12) and Fig.2, it can be seen that the angular swing amplitude can reach $7^{\circ}$ and the linear motion interference amplitude can reach $1.2 \mathrm{~m} / \mathrm{s}$, which means that 
the interference caused by flurry is strong, using the alignment algorithm presented in section $\mathrm{V}$, the initial alignment can be perform well, Fig. 3 shows the alignment error.
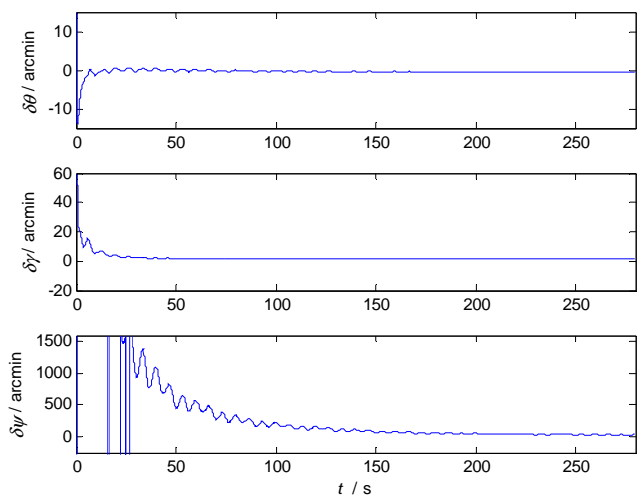

Figure 3. alignment error of self-alignment algorithm

Fig.3 illustrates that the alignment time of two level angles, roll and pitch is less than 30 seconds, the alignment time of yaw angles is about 150 seconds, in accuracy of estimation, the stable value of roll, pitch and yaw angle error are $0.51^{\prime}, 0.23^{\prime}$ and $6.9^{\prime}$ respectively, which shows that the presented algorithm can achieve alignment rapidly and accurately, this is because the presented alignment algorithm remove the linear motion interference fully by obtaining the equivalent specific force outputs at the shake center on the basis of suppressing the angular swing interference.

\section{SUMMARY AND CONCLUSIONS}

The angular wing and the linear motion interference caused by flurry are the main error sources of selfalignment, using the attitude update in inertial frame to record the attitude changes caused by sway interrupt, and translating the alignment problem into the Wahba problem can eliminate the effects of angular wing; using the characteristics that the body exists a shake center whose speed is zero, by acquiring the equivalent specific force of the shake center, the linear movement interrupting can be removed, for the angular wing and the linear motion interference are both removed, the presented algorithm can achieve alignment rapidly and accurately.

\section{REFERENCES}

[1] Y Y Qin, G M Yan, D Q Gu "A clever way of SINS coarse alignment despite rocking ship". Journal of Northwestern Polytechnical University, vol. 23, pp. 681-684,2005.

[2] D Q Gu, N El-Sheimy, T Hassan, “Coarse alignment for marine SINS using gravity in the inertial frame as a reference",Position,Location and Navigation Symposium,2008 IEEE/ION, Monterey,USA,May . pp.5-8,2008.

[3] C S Zhao, Y Y Qin, L Wei,“A Gravity-Based Anti-Interference Coarse Alignment Algorithm", Journal of Astronautics,vol.31,pp. 2335-2339,2010.
[4] F Sun, W Suh,"Research on coarse alignment of rotary SINS on a swing base" Chinese Journal of Scientific Instrument vol.31,pp.929936,2010

[5] F Sun, W Sun, "Research on mooring alignment with digital filter". Control and Decision, vol.25,pp.1870-1875, 2010

[6] J X Lian,Y G Tang, M P Wu,"Study on SINS alignment algorithm with inertial frame for swaying bases".Journal of National University of Defense Technology, vol.29,pp.95-99, 2007.

[7] Y $\mathrm{T} \mathrm{Mu}, \mathrm{H}$ T Fang, "A self-alignment method on large swing situations”. Science China Press, vol.42,pp.1364-1378, 2012.

[8] Y H Liu, X S Huang, D X Yue,"Lifting Wavelet De-Noising for SINS Alignment", Proceedings of the 2008 IEEE International Conference on Information and Automation, Zhangjiajie,pp.831834,2008 .

[9] Y G Wang, J S Yang, B Yang,"SINS initial alignment of swaying base under geographic latitude uncertainty". Acta Aeronautica et Astronautica Sinica, vol.33,pp. 2322-2329,2012.

[10] G Wahba,"A least squares estimate of spacecraft attitude”. SIAM Review, vol. 7,pp.409-411,1965.

[11] Yiğiter Yüksel,"Design and analysis of transfer alignment algorithms". Middle East Technical University, pp. 35-38,2005.

[12] Y Huang, J Q Han,"Second order ESO design and its stability analysis".Proc of IEEE Hong Kong Symposium on Robotics and Control. Hong Kong, pp. 128-133,1999. 